\title{
Mujeres, poder y comunalidad: testimonio oral de Marcelina Bautista Bautista, primera mujer agente municipal de la Región de San Andrés Chicahuaxtla, Oaxaca, México
}

\section{Women, power and communality: life story of Marcelina Bautista Bautista, first woman municipal agent of the San Andrés Chicahuaxtla Region, Oaxaca, Mexico}

\author{
LEONOR TERESO RAMÍREZ \\ Docente investigadora de la Facultad de Trabajo Social. Universidad Autónoma de Sinaloa, México \\ leonorteresoramirez@hotmail.com ORCID:0000-0003-1511-5815 \\ MARCOS SANDOVAL CRUZ \\ Promotor cultural Nanj niî́n-Triqui, Chicahuaxtla, Oaxaca \\ yioli15@gmail.com
}

\begin{abstract}
Resumen: El objetivo de la investigación es comprender el acceso a espacios políticos de las mujeres indígenas mediante, el testimonio oral de la primera mujer agente municipal de la Región Triqui Alta, Oaxaca, México. Se traen como referencia teórica los conceptos de poder, comunalidad y resistencias, así mismo se posiciona la crítica desde una lectura del feminismo comunitario, que reconoce los procesos por los cuales las mujeres indígenas resisten las múltiples opresiones derivadas de diversas intersecciones como clase, etnia y género. El diseño de la investigación es exploratorio-descriptivo, utilizando un enfoque cualitativo basado en el método de casos que recupera el testimonio oral de la primera mujer agente municipal de la región Triqui Alta en Oaxaca México. Una de las características de las comunidades indígenas es que rigen por el sistemas de usos y costumbres en donde la asamblea es el máximo órgano para la toma de decisiones, siendo en 1992 cuando se decidió que fuera Marcelina la primera mujer Agente Municipal de Santa Cruz Progreso, uno de los catorce pueblos que pertenecen a la Región de San Andrés Chicahuaxtla. Para Marcelina, estar en un espacio de poder desarrollando roles asignados culturalmente a hombres, negociando con toda la comunidad y realizando gestiones sociales para el bien común, ha sido un proceso difícil pero que a su vez deja claro la capacidad de mujeres de ocupar estos puestos que les han sido negados por mucho tiempo.
\end{abstract}

Palabras claves: Comunalidad, Poder, Feminismo comunitario, género, mujeres indígenas.

Abstract: The objective of the research is to understand the access to political spaces of indigenous women, through the oral history of the first female municipal agent of the Triqui Alta Region, Oaxaca, 
México. The concepts of power, communality and resistance are brought as a theoretical reference, likewise we position the criticism from a reading of community feminism, which recognizes the processes by which indigenous women resist the multiple oppressions derived from various intersections such as class, ethnicity and gender. The research design is exploratory-descriptive, using a qualitative approach based on the case method that recovers the Oral history of the first female municipal agent of the Triqui Alta region in Oaxaca, Mexico. One of the characteristics of indigenous communities is that they govern by the system of uses and customs where the assembly is the highest decision-making body and, it was in 1992 that it was decided that Marcelina would be the first woman Municipal Agent of Santa Cruz Progreso, one of the fourteen towns that belong to the San Andrés Chicahuaxtla Region. For Marcelina, being in a space of power developing roles assigned culturally to men, negotiating with the entire community and carrying out social efforts for the common good, has been a difficult process but which in turn makes clear the ability of women to occupy these positions that have been denied them for a long time.

Keywords: Communality, Power, Community Feminism, gender, indigenous women.

\section{INTRODUCCIÓN: TEJIENDO COMUNALIDAD EN CONTEXTOS COMUNITARIOS INDÍGENAS}

Oaxaca tiene una población total de 3967 889, es el estado con mayor porcentaje de personas indígenas (1 205 886) de acuerdo al total de su población y representa el $16.3 \%$ del total nacional, de acuerdo con datos de Instituto Nacional de Estadística, Geografía e Informática (INEGI, 2015), además, del 1.2 millones de personas de 3 años y más que hablan alguna lengua indígena, $637,196(52.8 \%)$ son mujeres y $568,960(47.2 \%)$ son hombres.

Asimismo, Oaxaca tiene 570 municipios, de los cuales en 245 municipios es en donde más del 40\% de la población son hablantes de lengua indígena (INEGI, 2015). De acuerdo con el Sistema de Información Cultural del Estado de Oaxaca hay 13 pueblos indígenas que son: Mazatecos, Zapotecos, Mixtecos, Triquis, Huaves, Chontales, Ixcatecos, Chocholtecos, Tacuates, Chatinos, Cuicatecos, Mixes y Chinantencos. Sin embargo, también están los pueblos Amuzgo y Zoque que no aparecen en dicha lista.

Con el anterior encuadre de datos que dibujan el panorama indígena en Oaxaca y la riqueza de pueblos y comunidades indígenas, se considera que sus cotidianidades parecen tener ciertas características y valores que se preservan desde tiempos antiguos y que se siguen transmitiendo a las nuevas generaciones, siendo uno de ellos el amor a la tierra/naturaleza y desarrollando en sus habitantes vínculo territorial y apego, elementos que permiten ir tejiendo comunalidad. Dicha comunalidad es tejida considerando a todas las personas que habitan la comunidad, creándose así lazos de parentesco que se hacen presente en el saludo, de esta forma las per- sonas pasan a ser tíos, tías, abuelos, abuelas, hermanos y hermanas de todos y todas.

Ya por sí mismo el concepto de comunidad manifiesta que no solo se trata de un espacio geográfico, sino de un conjunto de interrelaciones entre todas las personas que se consideran parte de ella y que trazan metas y objetivos comunes. Por otra parte, el concepto de comunalidad es más profundo aún, porque implica una cosmovisión donde intervienen elementos naturales, sociales y culturales. La comunalidad se construye día a día, se transmite mediante aprendizajes significativos que ayudan en la preservación de las pautas culturales propias de la comunidad.

La comunalidad está constituida por tres elementos: una estructura, que hace referencia al tejido social, costumbres, tradiciones y la geografía de su territorio; una forma de organización social, distinguida por la asamblea comunitaria en donde se toman todos los acuerdos junto con el sistema de cargos; y, una mentalidad que parte de su propia cosmovisión en el que hombres, mujeres y naturaleza comparte un territorio en común donde debe preservarse la paz y el respeto. Esta mentalidad colectiva es el elemento a partir del cual las diferentes sociedades originarias han dado forma a su estructura y organización en los distintos momentos de su historia. A su vez, esa mentalidad comunal es confrontada actualmente por el individualismo (Maldonado, 2015), un individualismo motivado por el neoliberalismo y los rasgo de colonialidad persistentes en las prácticas y discursos sociopolíticos.

La comunalidad es una forma de vida que implica una continua solidaridad y respeto entre los miembros y hacia todos los elementos que componen su contexto. Implica la 
enseñanza de valores fundamentados en el cuidado de la madre tierra, dadora de vida, fuente de alimento y cobijo en tiempos de resistencias y luchas. Un aspecto importante a resaltar es que la comunalidad no se reduce al espacio comunitario, sino que se enlaza con las personas que tuvieron que salir de la misma, sea por cuestiones laborales, familiares, personales, profesionales u otros, pero que conservan su identidad y luchan contra los procesos que intentan mestizarlos. Precisamente el concepto de zonas fronterizas dibuja la fuerza del vínculo territorial.

La comunalidad tiene cuatro elementos fundamentales y distintivos: el poder comunal, el trabajo comunal, la fiesta comunal, el territorio comunal (Rendón, 2004; Maldonado, 2015). El poder comunal como primer elemento, consiste en dos órganos de toma de decisiones, el más importante es la asamblea, en la cual participa toda la comunidad y es realizado cada que se quiere consultar sobre un tema/problema o cuando hay dudas sobre algún aspecto. Debido a que participan tanto ancianos como jóvenes la asamblea se hace en la lengua originaria, también como una oportunidad para preservarla.

El trabajo comunal, que es el segundo elemento, se basa principalmente en el tequio o trabajo que todo habitante debe dar para mantener en orden y para beneficio de la comunidad, por lo tanto es moralmente obligatorio. También se da la ayuda mutua en el que todas las personas se apoyan entre sí, sobre todo cuando se presenta alguna adversidad. Mientras tanto, la fiesta comunal como tercer elemento, son celebraciones religiosas y patronales, que generan un cúmulo de expresiones de identidad en varios aspectos reunidos, como la música, danza, vestido, gastronomía, lengua y otros elementos.

Finalmente, el cuarto elemento, el territorio comunal tiene como representantes a las autoridades agrarias y, se llama comunal porque las tierras no están dadas de forma individual por el gobierno, sino que las tierras le pertenecen a la comunidad y son las autoridades quienes las dividen y reconocen a las familias a quienes son otorgadas, incluso la comunidad puede desposeer de las tierras a los propietarios en caso de incumplimiento de deberes (Rendón, 2004; Maldonado, 2015). Por lo anterior, se considera que la colectividad es la que permite la continuidad del autogobierno mediante el sistema de usos y costumbres en las comunidades y pueblos originarios.

Sin embargo, no se puede generalizar que en todos los pueblos y comunidades indígenas se dé de la misma forma la posesión de tierras, ya que habría que analizarse la historia de cada uno y con ello interpretar si han cambiado las forma de organización social, por ejemplo en el pueblo Triqui los lazos parentales y la ocupación del territorio no se conciben de manera independiente sino que existe una forma organizacional de ocupar el territorio y una forma preestablecida para tener acceso a las tierras y que a su vez condiciona las relaciones sociales, especialmente aquellas que conducen al matrimonio (Lewin y Sandoval, 2007). En esta lógica, por una parte, hay acuerdos entre linajes o grupos de socios para el matrimonio y que a su vez seguirá determinando la herencia, posesión o propiedad de tierras que han sido dados desde la colonia, por otra parte, el cuidado y defensa del territorio se hace de manera colectiva.

\section{METODOLOGÍA}

El presente estudio es exploratorio-descriptivo con enfoque cualitativo. Se trata de un estudio de caso de la región Triqui alta, Oaxaca, México. El estudio de caso posee una doble connotación, por una parte porque el contexto es una región específica que comprende 14 pueblos y se llama San Andrés Chicahuaxtla, por otra parte, se trata de una entrevista a un solo caso representativo para entender procesos políticos de las mujeres en dicha región. Se justifica porque la persona entrevistada tiene características únicas, es atípico en relación a otros casos, y ello obliga a profundizar en su análisis, en este caso la entrevistada fue Marcelina Bautista Bautista (MBB).

Los casos atípicos suelen ser sobre algo específico, que funciona para explicar una realidad más compleja, muchas veces el caso viene dado, "no interesa porque con su estudio se aprenda sobre otros casos o sobre algún problema general, sino porque se necesita aprender sobre ese caso particular" (Stake, 1998, p. 16). Se sitúa entonces el estudio en la historia Marcelina Bautista Bautista quien fue la primera mujer Agente Municipal de la Región. Para ello, se utiliza la técnica de observación y el testimonio oral que deviene de las historias orales, entendiéndose esta última como:

Un espacio de confluencia interdisciplinaria, que al surgir desde el seno de la historia social procede a seleccionar nuevos sujetos sociales, en escalas y niveles locales y regionales; con el fin de abordar fenómenos y cuerpos de evidencias específicas y controlables; con técnicas precisas y fuentes nuevas y plurales, que tiene el propósito de lograr aproximaciones cualitativas de los procesos y fenómenos sociales e individuales [...] por destacar y centrar su análisis en la visión y versión que desde dentro y lo más profundo de la experiencia, expresan los sujetos sociales considerados centralmente en el ámbito de la historia social-local-oral (Aceves, 1993, p. 234). 
Así mismo, la elaboración del artículo se hace desde una lectura feminista, dado que su importancia radica en contar historia poca o casi nunca contada, para visibilizar otros espacios, otros tiempos y a otras memorias. Además los testimonios orales sobre la propia historia y experiencias situadas-particulares permitirán a otras mujeres visionarse en espacios políticos en la posteridad.

En este sentido, algunos datos biográficos de la maestra Marcelina Bautista Bautista son: nació en la comunidad de Santa Cruz Progreso, Chicahuaxtla, Oaxaca en 1956, hija de Ramón Bautista y Casimira Bautista, quienes tuvieron únicamente dos hijas, entre ellas, Marcelina. La maestra estudió el nivel básico y su ocupación fue profesora de educación primaria, actualmente jubilada y dedicada a trabajar sus tierras y cuidar su ganado. Tiene cinco hijos, dos hombres y tres mujeres, en cuanto a su situación civil, es viuda.

\section{RESULTADOS Y DISCUSIÓN: MUJERES, PODER Y COMUNALIDAD EN LA TRIQUI ALTA, OAXACA}

Oaxaca tiene ocho regiones que concentran un gran número de hablantes de lengua indígena estos son: Valles Centrales, Costa, Sierra Norte, Sierra Sur, Cañada, Mixteca, Istmo y Papaloapan. No obstante, usar esta referencia niega la existencia de los Pueblos Indígenas de Oaxaca, es una regionalización hasta etnocida, bastante absurda, el mejor ejemplo de esto es la región Triqui, que está dividido en la mixteca, sierra sur, con pertenencia a municipios mestizos como Putla, Juxtlahuaca, Constancia del Rosario Tlaxiaco y no se nombra como un pueblo Triqui con raíces históricas y culturales comunes y con formas de organización distintas que lo hacen único en su devenir histórico.

Por lo anterior situamos el estudio considerando a San Andrés Chicahuaxtla como una región en sí misma, es una comunidad perteneciente al municipio de Putla Villa de Guerrero, Oaxaca, es también conocida como Triqui alta y comprende actualmente catorce pueblos, que son: San Andrés Chicahuaxtla (Yuma' Niko' en lengua Nanj Ni'în que significa en español Pueblo Grande, debido a que de los catorce pueblos es el más grande); Santa Cruz Progreso; Llano de Zaragoza; Yosonduchi; San Marcos Mesoncito; Miguel Hidalgo; San Gabriel; La Cañada Tejocote; Joya Grande; Plan de Guajolote: Los Reyes Chicahuaxtla; La Laguna Guadalupe; San Isidro de Morelos y, San Antonio dos Caminos.

En estos catorce pueblos que comprenden la Triqui Alta, se utiliza el sistema de usos y costumbres, por lo que hay sistema de cargos que ocupan regularmente hombres, mismos que son nombrados mediante asamblea comunitaria.
Las mujeres que llegan a ocupar cargos son principalmente solteras, viudas o divorciadas/separadas y los cargos son de menor jerarquía. Las actividades de los cargos ocupados por las mujeres obedecen de cierta forma a la ampliación de trabajos domésticos-privados al espacio público. Mientras tanto, las mujeres casadas no ocupan cargos debido a que sus esposos ocupan cargos y con ello también las representan.

En esta lógica, los hombres son quienes ocupan cargos de mayor responsabilidad ya sea en la agencia municipal, la sindicatura y la tesorería. Las ideas culturalmente establecidas en torno a los estereotipos sobre la masculinidad y la feminidad han normalizado que los hombres son los negociadores, saben tomar mando, decisiones y dar órdenes, mientras que las mujeres deben hacer labores de acuerdo a su naturaleza, misma que es en el hogar o en actividades muchas veces desvalorizadas y no debido a que no es costumbre que una mujer quiera mandar al pueblo (Bonfil y Martínez, 2003).

Como se ha mencionado anteriormente, el sistema de usos y costumbres tiene como máximo órgano decisor a la asamblea comunitaria, es decir, se reúne a todo el pueblo y se nombra a quienes tomarán los cargos año con año. En este escenario, es donde se genera un parte aguas cuando la asamblea de uno de los pueblos, Santa Cruz Progreso, en el periodo comprendido entre 1991-1992 le otorga el cargo de agente municipal a la primera mujer, la maestra Marcelina Bautista Bautista, a la edad de 35 años. Ninguno de los otros trece pueblos lo había hecho, ni siquiera en la actualidad el pueblo más grande, San Andrés Chicahuaxtla ha nombrado a ninguna mujer en el cargo más importante, el de agente municipal. Por ello, la experiencia de Marcelina resulta enriquecedora y permite analizar el papel de las mujeres indígenas en los cargos comunitarios, así como tambien, ofrece un panorama de las dificultades que atraviesan para que la comunidad confíe y trabaje en conjunto con ellas. Así comienza a contar su historia, la que orgullosamente fue la primera mujer en ocupar un alto cargo político-comunitario:

Más que nada, pues este pueblito de Santa Cruz apenas fue de nueva creación y habían puros hombres ocupando el cargo de agente $y$, en una asamblea eligen nueva autoridad y entonces, ni yo misma me di cuenta de cómo me eligieron, porque no había pasado por ningún cargo ni una vez, ni policía, ni sindico, ni regidor, nada pues, sino que el primer trancazo que me dieron, porque era algo nuevo, yo así sentí medio raro, me sentí penosa, y pues me dijeron, no pues ni modo tienes que venir, tienes que pasar el cargo, y pues ni modo, porque la mayoría no me quisieron cambiar por otra gente, y bueno, bien 
o mal pensé que a la primera me sale mal, a lo mejor a la segunda pues ahí me va bien y no, resulta que ese año no me quisieron cambiar, no me dejaron renunciar y vine y ocupe mi cargo (MBB).

El acceso de las mujeres indígenas a espacios políticos ha sido lento, persiste la idea de la superioridad de los hombres en cuanto a que culturalmente se ha construido la idea de que ellos son protagonistas del espacio público, mientras que las mujeres están destinadas al trabajo de la reproducción. Por otra parte, la ocupación de cargos por el sistema de usos y costumbres no ha sido relevante en los estudios ni ha habido mucha preocupación por documentarlos a pesar de que en el estado de Oaxaca la Constitución estatal incluye el sistema de usos y costumbres como vía para nombrar autoridades municipales: de los 570 municipios, 140 eligen autoridades municipales por la vía de elecciones y partidos, y 450 por la de Usos y Costumbres (Velásquez, 2003).

Las gentes se extrañaron pues, por que como una mujer iba a ocupar ese puesto de agente municipal. Yo no me sentía preparada, pues haga usted de cuenta que pues si ya pase algún cargo, dos cargos ahí en la agencia, aunque sea topil (policía) que dijéramos no, pues por lo menos si sabe uno que hacer, pero no, era mi primera vez, pero aun así no tuve problemas, toda la gente me obedecían y pues cualquier trabajito que hay, trabajaban unidos (MBB).

Ciertamente, es el espacio de la organización del poder público, en donde la participación de las mujeres es la más baja, aunque no por ello es nula más bien es invisibilizada o poco reconocida. La escasa participación de las mujeres indígenas en cargos de representación política está relacionada no sólo con las dificultades de los hombres para compartir el poder, sino al hecho de que las mujeres no tienen el nivel de escolaridad o las habilidades para desempeñar las funciones que el Estado espera de la representación política (Patrón, 2015). Sin embargo, los hombres aunque no tengan tampoco altos niveles de escolaridad, pocas veces son juzgados, al contrario, se confía en ellos.

Yo pensé que no iba a poder con el cargo, pero pues no hubo conflicto y con pena y todo así lo hice. La gente se sorprende cuando las mujeres ocupan cargos porque se tiene la idea que las mujeres solo servimos para la casa y los hijos y los hombres son lo que saben tomar decisiones y dirigir, pero yo digo que es bueno que las mujeres tengan cargos más altos porque aunque es extraño para algunas personas que las mujeres ocupen esos cargos, pero ahora ya sabemos que hombres y mujeres somos iguales y podemos hacer de todo (MBB).

Al respecto, la diferencia entre hombres y mujeres es que la participación femenina en los cargos de decisión no pasa necesariamente por el filtro de la obligatoriedad y en la mayoría de los casos, debido a su rol reproductivo y al estereotipo de género, las mujeres son nombradas en cargos relacionados con la salud y la educación, precisamente los campos de mayor incidencia de las políticas del gobierno trazadas para las mujeres, por lo que ellas son regularmente las administradoras de los bienes que el Estado ofrece a la sociedad. Adicionalmente, otro factor que influye en la aceptación y convocatoria de las mujeres en las decisiones públicas es su estatus marital, su edad y su escolaridad (Vázquez, 2011).

Yo nada más termine sexto año de primaria y empecé a trabajar de maestra y solo íbamos a Oaxaca la capital o Tlaxiaco a estudiar y tomar cursos de capacitación y con eso salíamos con el título de una escuela como si fuera normalista para nivel primaria. Mi trabajo como maestra me ayudó mucho porque quienes tenemos un cargo comunal no recibimos ningún pago, entonces mi salario me ayudó mucho para pagar los gastos, porque todo sale de nuestro bolsillo, por ejemplo para ir a gestionar recursos al municipio o las instituciones (MBB).

En el sistema de cargos el papel de la mujer es importante, como esposa de quien tiene el cargo; ella debe preparar, con otras mujeres, las comidas y arreglos religiosos correspondientes, pero ¿qué sucede cuando las mujeres ocupan el cargo de agentes municipales? ¿Quién desempeña las actividades que realizaban las esposas? (Barrera-Bassols, 2006), resulta que en estos casos, la mujer que es agente municipal y como el caso de Marcelina, una mujer viuda, ella se tiene que responsabilizar de todo el trabajo que ello implica tanto del cargo ocupado como de las actividades domésticas, de cuidado y comunitarias.

Luego en donde también se fija mucho la gente si de verdad sabemos trabajar es cuanto se tienen que hacer fiestas que son importantes para el pueblo, el organizarlos y que todo salga bien. Por ejemplo, cuando es la fiesta de carnaval una se tiene que apurar (...) yo, un poco iba a hacer trabajo de mujeres que era hacer tortillas $y$ comida y después me iba a hacer el trabajo que hacen 
regularmente los hombres, pues como agente tenía que hacerlo, como por ejemplo, cuando los que se disfrazan en el carnaval (chilolos, así conocidos en la comunidad) van de casa en casa, pues yo tengo que ir a vigilar porque si hay algo, un problema o algo pues es responsabilidad mía y de todo mi comité (MBB).

Por otra parte, a pesar de la participación de las mujeres en algunos cargos u otras actividades por ser esposa del hombre que tiene cargo, la responsabilidad doméstica sigue siendo la misma. La carga de trabajo femenino, en sus roles de reproducción y producción limita la libertad de las mujeres para acumular experiencia y desempeñar esos cargos. Los casos excepcionales muestran que se trata de mujeres que han asistido a la escuela; mujeres con capacidad de liderazgo según los parámetros que los propios hombres consideran; mujeres sin hijos o mujeres que han dejado atrás su etapa reproductiva, con la finalidad de que tengan el tiempo suficiente para atender su cargo (Patrón, 2015: p.56).

Las mujeres que ocupan cargo son solo las solteras y viudas, las casadas no porque las representa su marido, si el esposo de alguna mujer ya cumple con todos los cargos comunitarios pues a la esposa ya no le corresponde porque ella está dedicada a su familia y a su casa. Mientras que las solteras y viudas no hay quien las represente y tienen que cumplir con los cargos que le corresponde a toda persona adulta, así es como funciona el orden de la comunidad (MBB).

Vázquez (2011, citando a Hernández y López, 2006) considera que en la construcción de la ciudadanía bajo el sistema de usos y costumbres interviene el género como factor limitante, porque la identidad comunitaria está dada por la posesión de la tierra y las mujeres al no heredarla directamente, sino mediante su esposo quien asume el papel de jefe de familia, tiene un estatus incompleto de ciudadanía y por ello se justifica y no se les obliga a desempeñar cargos comunitarios. En esta lógica, se podría entender que, las mujeres de cierta forma ceden parte de su ciudadanía a los hombres con los que conviven, a veces a los padres, a veces a los esposos y otras tantas a los hijos. Asimismo ven en el papel en este caso el de agente municipal un pater estado, es decir, como un padre de familia que está al pendiente y cuida de su familia, la comunidad, y por eso solo visualizan en ese cargo a los hombres, los que proveen y los que la cuidan. No obstante, consideramos desde la postura feminista comunitaria que la ciudadanía no puede determinarse solamente con la propie- dad de la tierra, debido a que las mujeres al tener un rol relevante en el desarrollo comunitario y volverse actoras con voz en las asambleas comunitarias demuestra su ciudadanía.

Después de ser agente ocupe otros cargos como secretaria del agente y otro año fui tesorera aunque en esos cargos ya es menos, porque quien dirigen es quien manda, es como el papa que dice que se tiene que hacer $(\mathrm{MBB})$.

En esta lógica, si bien, la familia constituye un espacio de reproducción de la desigualdad de género (Ariza y Oliveira, 2005) aun así, tambien la familia entendida en su rol más amplia, como familia comunitaria donde se tejen lazos de parentesco que van más allá de los lazos sanguíneos, es tambien la base donde se sientan las bases de solidaridad entre mujeres y hombres y, por lo tanto, todas las personas se vuelven parte de la complementariedad y dualidad hombre/ mujer-naturaleza o bien cuerpo-territorio.

No obstante, no se puede dejar de visualizar que debido a que prácticamente todas las actividades domésticas son desvalorizadas e invisibilizadas, y recaen además sobre las mujeres, esto las obliga a cumplir con dichas tareas por considerarlas natural a su género y es así como Marcelina expresa que realizaba doble e incluso triple jornada laboral, ninguna no remunerada, por una parte, asumía la jefatura de su familia y cuidaba de sus hijas y por otra parte asumía lo que implica ser agente municipal y cumplir con los tequios o trabajos comunitarios:

Yo soy jubilada desde hace 15 años y ya no tengo hijos pequeños, así que ya solo me dedico a descansar. Pero en aquellos tiempos en que yo tenía cargo mis hijos estaban pequeños fue muy difícil porque estudiaban y tenía yo que salir y cubrir el gasto para mis hijos. Mas a parte el trabajo comunitario de los tequios, tenemos que ir y lo que hacemos depende de la autoridad en turno, por ejemplo si se tienen que arreglar la calle, limpieza en la carretera, limpieza en las escuelas, limpiar los límites de nuestro pueblo con otro pueblo u otras cosas (MBB).

Es así, como a las mujeres se les asignas roles obligatorios que por su condición de género, según las construcciones culturales deben realizar, mismas que tambien se amplían al espacio público, incluso Vázquez (2011) asegura que la salud y la educación, son precisamente los campos de mayor incidencia de las políticas de gobierno trazadas para las mujeres y así lo expresa Marcelina: "las mujeres ocupan regularmen- 
te el cargo de comité de la escuela primaria, y los hombres ocupan el cargo de policías comunitario, porque ese cargo necesita fuerza y eso es de los hombres, para detener a quien comete algún delito o también porque los policías necesitan quedarse toda la noche haciendo guardia, por eso las mujeres no porque es peligroso".

Cuando ocurren las excepciones en que mujeres ocupan cargo, se enfrentan a diversos obstáculos que se materializan en los procesos de toma de decisiones, gestiones en instancias lideradas por hombres, rol de mando, entre otras, debido a que no se les da el mismo valor y confianza, sin embargo, es necesario decir que hay mujeres que se sobreponen a ello y trabajan tal como lo dice Marcelina:

El trabajo que más hacemos es hacer gestiones, porque en aquel tiempo no hay apoyos de gobierno, todo salía de la bolsa de una. Cuando yo iba a gestionar recursos me acompañaba a veces el suplente o secretario o a veces iba yo sola, a veces se consigue o a veces no. Sobre todo siendo mujer muchas veces no nos hacen caso los funcionarios. El agente de los municipios a donde iba a gestionar me trataba un poquito diferente, pero también es parte de la política, por ejemplo en las campañas prometen muchas cosas, pero a la mera hora no dan nada $(\mathrm{MBB})$.

Lo anterior por lo que se ha venido argumentando, que la política pertenece a los varones al igual que les pertenece la racionalidad, la jerarquía, la cultura, el temple, el valor, el carácter y el acuerdo, mientras a las mujeres se les excluye de la política y se les pide limitarse al buen manejo del hogar, la obediencia, la dulzura y crear el ambiente adecuado para la tranquilidad y éxito de los varones (Valcárcel, 1995). Aunado a esto, cuando las mujeres ocupan un cargo y no rinden las cuentas claras y reportan trabajo, se les desestima y con ello se refuerza desde el sistema patriarcal la imposibilidad de las mujeres al frente de los puestos de mayor estatus.

Aun cuando sucede lo contrario y las mujeres demuestran su capacidad de gestión, de organización y logran cosas importantes para sus comunidades, es raro que se les dé el reconocimiento a ellas. Lamentablemente, aun cuando se den casos de éxito, el hecho de nombrar a otras mujeres de forma consecutiva es difícil, sea por que las mujeres no se atreven a tener protagonismo y mostrar sus capacidades, porque sus esposos no se los permiten o porque son acuerdos comunitarios.

Todas las autoridades cuando están en el periodo que les corresponde tienen que realizar un trabajo, una obra que beneficie a la comunidad. Lo que hice en mi periodo de agente, fue terminar la construcción de la tienda de la Compañía Nacional de Subsistencias Populares (CONASUPO), porque los que estuvieron antes de mi pusieron el cimiento. $Y$ ya los que estaban conmigo nos organizamos y lo terminamos, también otros trabajitos que hay este, bueno donde hace falta, principalmente la barda donde iba a estar la iglesia y otros trabajitos que hay en la comunidad.

Se tiene que hacer asambleas con todo el pueblo donde salen acuerdos de lo que se tiene que hacer, y aunque con pena y todo pero yo tenía que dirigir las asambleas y aprendí y ahora sí que mi obligación es cumplir y hacer lo que se puede. Yo tenía suplente hombre, todo mi equipo eran hombres, yo soy la única mujer que vino, pero si me hacían caso, trabajamos bien, en aquel tiempo eran más respetuosos y la comunidad estaba contenta con lo que se había hecho, la gente era muy trabajadora y este pues sí, la gente estaba contenta. Después de mi siguieron viniendo hombres en el cargo de agente. Pero el pueblo más grande de la región es Chicahuaxtla pero ninguna mujer ha venido en el cargo de agente en ese pueblo (MBB).

Incluso la misma maestra Marcelina comenta que si le pidieran ser de nuevo agente municipal, no aceptaría, debido a la edad que ahora tiene, y porque se dedica a otras actividades como sembrar sus tierras para autoconsumo, cuidar de su ganado y tejer sus huipiles. La maestra es jubilada y de lo que si tiene una visión clara es que le gustaría que otras mujeres ocuparan puestos importantes es su región, sobre todo porque ahora hay mujeres preparadas profesionalmente.

\section{CONCLUSIONES}

En los movimientos feministas comunitarios, una de las ideas que se debaten es si en los pueblos originarios y afrodescendientes la división sexual del trabajo se dio de la misma forma que en Occidente, la idea central gira alrededor de explicar que en el caso de estas comunidades propias de Latinoamérica, las mujeres participaban activamente en el espacio público desde sus mismas fundaciones, ya sea en la agricultura, la ganadería, en la comunalidad misma y todo lo que implica. Además, en los pueblos originarios la feminidad era considerada sagrada, debido a que a la madre tierra se le ofrendaba lo mejor porque es en su seno de donde viene todo lo que hace posible la vida, de ahí se alimenta ella misma, de ahí se alimentan los animales, de ahí se alimentan las 
personas, por ello la cosmovisión desde la dualidad hombre/ mujer-naturaleza. Sin embargo, es necesario seguir estudiando qué tanto influyó la imposición de un sistema patriarcal, colonizadora, heredada desde tiempos de la conquista y que ha dejado marcada la brecha de desigualdad entre mujeres y hombres en una tajante división de roles que se visibiliza en espacios como el político.

Por otra parte, cabe decir que el reconocimiento legal de los usos y costumbres de los pueblos y comunidades indígenas en la actualidad, es un paso importante y democrático que representa además, el goce de los derechos y libertades del que toda persona debe gozar, sin embargo, también se reconoce que es necesario generar estrategias al interior de estas para que las mujeres también se vuelvan protagonistas de espacios políticos que beneficiaran a las propias comunidades, ya que al integrar a hombres y mujeres a estos espacios se puede tener un visión más integral no solo de los problemas, sino de los beneficios que pueden tener, en diversos aspectos.

Para que lo anterior pueda ser factible, es necesario sensibilizar, concientizar y educar a las mujeres en temas de igualdad, para que ellas se autoconfronten y dialoguen de otras formas con otros espacios, tiempos y actores y se atrevan a ser protagonistas de espacios políticos posicionándose y tejiendo comunalidad desde sus saberes y experiencias. Asimismo se debe trabajar en las masculinidades positivas, responsables, que se motiven a realizar actividades que han estado culturalmente asignadas a las mujeres.

Un elemento a resaltar respecto al desarrollo social de los pueblos y comunidades es la movilidad de las personas pertenecientes a la comunidad, debido a que se siguen dando fenómenos como: la migración de hombres principalmente en busca de trabajo remunerado y la migración de jóvenes en busca de oportunidades de formación profesional. Esto obliga a la inserción de mujeres en cargos comunitarios donde van adquiriendo conocimientos y desarrollando capacidades y habilidades, se abren las oportunidades para que ellas ocupen otros cargos antes destinadas solo a hombres, como son el de agente, sindico/a y tesorero/a.

Finalmente, se considera que el desarrollo profesional y laboral de las juventudes que han migrado y retornan a trabajar por el bien común está provocando una reconfiguración de las mentalidades, debido a que en las asambleas comunitarias se escucha hablar de la igualdad de género, seleccionando a escrutadores secretarias y secretarios en cuotas iguales, aspecto que se debe aprovechar para formar a las nuevas generaciones en temas de igualdad y equidad de género.

\section{REFERENCIAS}

Aceves, J. (1993). Historia oral. México: Instituto Dr. José María Luis Mora

Ariza, M. y Oliveira, O. (2005). Cambios y continuidades en el trabajo, la familia y la condición de las mujeres. En: E. Urrutia. (Coord.) Estudios sobre las mujeres y relaciones de género en México: aportes desde diversas disciplinas. México: El Colegio de México, 43-86.

Barrera-Bassols, D. (2006). Mujeres indígenas en el sistema de representación de cargos de elección. El caso de Oaxaca. Agricultura, sociedad y desarrollo, 3(1), 19-37. Recuperado de: http://www.scielo.org.mx/pdf/asd/v3n1/ v3n1a2.pdf

Bonfil, P. y Martínez, E. (2003) Diagnóstico de la discriminación hacia las mujeres indígenas. México: Edit. Colección Mujeres Indígenas

Instituto Nacional de Estadística, Geografía e Informática (INEGI) (2015). Principales resultados de la Encuesta Intercensal 2015 Oaxaca. México. Recuperado de: http:// internet.contenidos.inegi.org. $\mathrm{mx} /$ contenidos/Productos/ prod serv/contenidos/espanol/bvinegi/productos/nueva estruc/inter censal/estados2015/702825079857.pdf

Lewin, P. y Sandoval, F. (2007). Triquis Pueblos indígenas del México contemporáneo. México: Comisión Nacional para el Desarrollo de los Pueblos Indígenas Recuperado de: https://www.gob.mx/cms/uploads/attachment/file/12593/ triquis.pdf

Maldonado, B. (2015). Perspectivas de la comunalidad en los pueblos indígenas de Oaxaca. Bajo el Volcán, (15)23, pp. 151-169. Benemérita Universidad Autónoma de Puebla, México Recuperado de: https://www.redalyc.org/ pdf/286/28643473009.pdf

Martínez, J. (2015). Conocimiento y comunalidad. Bajo el Volcán, 15(23), 99-112. Recuperado de: https://www.redalyc.org/pdf/286/28643473006.pdf

Paredes, J. (2013). Hilando fino desde el feminismo comunitario. México: Cooperativa el Rebozo, Zapateándole, Lente Flotante, En cortito que's palargo y AliFem AC. Recuperado de: https://sjlatinoamerica.files.wordpress. com/2013/06/paredes-julieta-hilando-fino-desde-el-feminismo-comunitario.pdf

Patrón, T., S. (2015). Participación política de las mujeres indígenas en los municipios que se rigen por usos y costumbres. El caso de san juan Cotzocón y Santiago Zacatepec, Oaxaca. Recuperado de: file:///C:/Users/GERARDO/ Downloads/20799-Texto\%20del\%20art\%C3\%ADCulo-46911-1-10-20150809.pdf 
Rendón, J., J. (2004). La comunalidad o modo de vida comunal de los pueblos indios. México: Dirección General de Culturas Populares e Indígenas.

Stake, R. (1998). Investigación con estudios de caso. Madrid: Morata. Recuperado de: https://www.uv.mx/rmipe/ files/2017/02/Investigacion-con-estudios-de-caso.pdf

Valcárcel, A. (1995). Feminismo y poder político. En C. Martínez. (Comp.) Feminismo, ciencia y transformación social. España: Universidad de Granada, 223-233. Recuperado de: https://valcarcelamelia.files.wordpress.com/2015/07/ feminismo-poder-y-politica.pdf

Vázquez, V. (2011) Usos y costumbres y ciudadanía femenina. Hablan las presidentas municipales de Oaxaca. 1996-
2010. México: Porrúa. http://biblioteca.diputados.gob. mx/janium/bv/ce/scpd/LXI/uso cost fem.pdf

Velásquez, C., M. (2003). ¿Mujeres indígenas gobernando municipios en Oaxaca? México Indígena, 2(5). Pp: 24-31.

*Agradecemos a la maestra Marcelina Bautista Bautista por permitirnos contar su historia y con ello discutir las tareas pendientes en materia de género, situar su experiencia en el ámbito público-político y permitir construir conocimientos que dignifican a los pueblos y comunidades indígenas. 